\title{
A NEW PARADIGM OF SECURITY MODEL FOR TREASURY INFORMATION SYSTEM -- E- GOVERNANCE
}

\author{
Ramineni Sivarama Prasad \\ Research Guide. Department of Computer Science \& Engineering., Acharya Nagarjuna \\ University, Guntur-Dt. AP-522510. \\ Gurram Veera Raghavaiah.,
}

Research Scholar in Department of Computer Science.,Reg. No: PP.COMP.SCI.0388 Rayalaseema

University., Kurnool. AP-518002.

\begin{abstract}
The world of Computing has been completing 80 years in applying scientific methodologies to understand basic principles of Emerging Technologies. In recent times, IT has great influence on how different Indian Government Departments operate. Security is one of the most important issues in E-governance projects. Egovernance applications will be increasingly used by the citizens of many countries to access a set of government services. Currently, the use of the E-government applications arises many challenges; one of these challenges is the security issues. E-government applications security is a very important characteristic that should be taken into account. This paper analyses about new secured model developed for Treasury Information System by using Quantum Key Distribution based verifiable and traceable cipher text attributes based encryption (QKD-VTCP ABE) algorithm that support an integrated internet-based E-governance applications.
\end{abstract}

Keywords: Indian treasury, Quantum key distribution, CP - ABE, Heisenberg's principle of uncertainty. Ditional security monitoring tools in Treasury Information System.

Cite this Article: Ramineni Sivarama Prasad and Gurram Veera Raghavaiah, a New Paradigm of Security Model for Treasury Information System -- E-Governance, International Journal of Computer Engineering and Technology, 10(2), 2019, pp. 169179.

http://iaeme.com/Home/issue/IJCET?Volume=10\&Issue=2 


\section{INTRODUCTION}

The Indian government adopted a Network and Information security measures as a part of eGovernance to protect the vital information's that got incorporated in the framework involving the e-Governance. The major challenges observed in the design of the e-governance system was found to be regarding risk involving the security of the data shared among the network. Therefore, it is necessary to address the issues regarding the security issues that are incorporated within an E-governance project as it comprises of critical information's that get transmitted through Intranet and LAN. Thus, for maintaining the confidential data, it is necessary to develop an appropriate model based on the treasury information system. The security is critical was found to be some significant challenges in the e-Governance to safeguard the assets and maintain the confidentiality of transactions and information on the network. Government documents such as online funds transfer, birth and death registration, vehicle license, passport applications, land documents etc. need to be protected from unauthorised users in e-Governance projects while incorporating the information systems. Hence security was found to be a critical aspect for the implementation of Treasury Information Systems on an integrated internet-based application. Furthermore, the majority of E-governance applications observed in India were found to be related to the applications on their Intranet. Thus, it is necessary to develop an appropriate integrated model devised in the form of an Integrated Internet-based system for providing the required in the by providing new security models on data and network transmission.

\section{AIM}

The research aims at designing a security system for protection of treasury information.

\section{OBJECTIVES:}

The research has the following objectives to achieve the aim as mentioned above:

1. To explore the various existing Treasury Information Systems (TIS) and its challenges of various governments

2. To find the better functioning of Treasury Information Systems across the worldwide

3. To develop a CP-ABE based security algorithm for obtaining security to the Treasury Information System.

4. To evaluate the performance of a new proposed secured Treasury Information System model and to compare it with existing techniques.

\section{PROPOSED METHODOLOGY}

Security is an important challenge in the Treasury Information System (TIS) model in-transmit over Local Area Network (LAN) and intranet. Also, government documents such as online funds transfer, birth and death registration, vehicle license, passport applications, land documents, etc. These documents have to protect from unauthorised users in e-Governance projects. Thus, security is the most critical issues in thee-Governance to safeguard the assets and maintain the confidentiality of transactions and information on the network. Therefore, the solution is that to develop a new high-security algorithm for Treasury Information Systems on an integrated internet-based application.

STEP 1: Obtain the information from the treasuries like workflow-based treasury payment/receipt transaction-processing system and perform the SWOT analysis. 


\section{Strength:}

Often, an organisation's strengths provide an easy opportunity for increasing their security, by building on or positively modifying existing controls, security can usually get increased.

\section{Weakness:}

Weakness can be specific or broad. A general lack of security program or culture is considered as the weakness but is not defined enough to guide action. OutLook on specific areas is necessary. Organizations fall prey to vulnerabilities that would not have got prevented if they had a proper patch management program. Implementing a patch management program involves spending money, but it is a small price compared to remediating damage caused by an exploited vulnerability.

\section{Opportunities:}

Opportunities are low hanging fruit over which you can take advantage. The best part is that taking advantage of most opportunities do not require management approval or any significant spending.

\section{Threats:}

Many threats, especially from a security perspective, are easy to delineate. If the organisation is subject regulations such as PCI DSS, HIPAA or SOX, the cost of non-compliance can be astronomical. The costs of reputational damage often outweigh the fines for non-compliance. And the fines for non-compliance are stiff.

A SWOT analysis is an essential test for implementing any plan. For acceptance of the plan, strength and opportunities should outweigh the weakness in the plan and possible threats that may arise on applying it.

STEP 2: From that data, need to pre-process the text using natural language machine learning to get effective information.

Pre-processing consists of four parts, they are Cleaning, Annotation, Normalisation and Analysis

\section{Cleaning:}

It consists of getting rid of less useful parts of the text through stopword removal, dealing with capitalisation and characters and other details.

Annotation:

It consists of the application of a scheme to texts. Annotations may include structural mark up and part-of-speech tagging.

\section{Normalisation:}

It consists of the translation (mapping) of terms in the scheme or linguistic reductions through Stemming, Lemmatization and other forms of standardisation.

Analysis:

It consists of statistically probing, manipulating and generalising from the dataset for feature analysis. 
STEP 3: Once the pre-processing is done, by developing a conceptual framework using Quantum Key Distribution based verifiable and traceable cypher text attributes based encryption (QKD-VTCP ABE) algorithm.

\subsection{Quantum Key Distribution (QKD)}

With the exponential growth of network bandwidth, computational resources and the popularity of the internet, cloud security has become one of the interesting research areas of cloud computing.

Cloud computing services are used widely in different applications such as medical, defence, education, health etc., for data storage and elastic computing. Attribute-based encryption is a public key encryption algorithm that allows cloud users to secure their sensitive information in the public cloud servers. Traditional attribute-based encryption models apply to standalone applications with limited resources constraints, in-secured key generation and slower processing speed. Also, users ${ }^{\text {ee }}$ data access control mechanisms must satisfy the process of protecting data from unauthorised users as well as from third-party providers. To overcome these issues a novel quantum key distribution (QKD) based cipher text-policy ABE model got implemented in the cloud environment.

Verifiable and Traceable Cipher-Attributes Based Encryption scheme (VTCP-ABE) simultaneously supports the properties of verifiable outsourced decryption and white-box traceability without compromising the physician's identity privacy.

STEP 4: When the QKD-VTCP ABE applied it first takes the text data and encode the information and key will get generated.

The scheme is for ciphering and deciphering the information for a secured transfer. Quantum Key distribution gets applied to verifiable and traceable Cipher which encrypts based on attributes. The information is encoded, and the key for decryption gets sent along with the encoded information. The generation of the key gets after encoding the data, and this key decrypts at the destination node.

STEP 5: Key gets sent to the cipher text, from that the text data will be decoded and generate key secret.

Cipher text receives the key, and the text data gets decoded and requires the key secret that gets used for decoding for analysis.

STEP 6: Once the decoding gets done, then from that generated key data we get exact text and compare efficiency with the existing algorithm.

After decoding the secret information, the text received gets compared with the original text. The comparison will show the efficiency of the existing algorithm.

Given below is the flow chart for the proposed system 


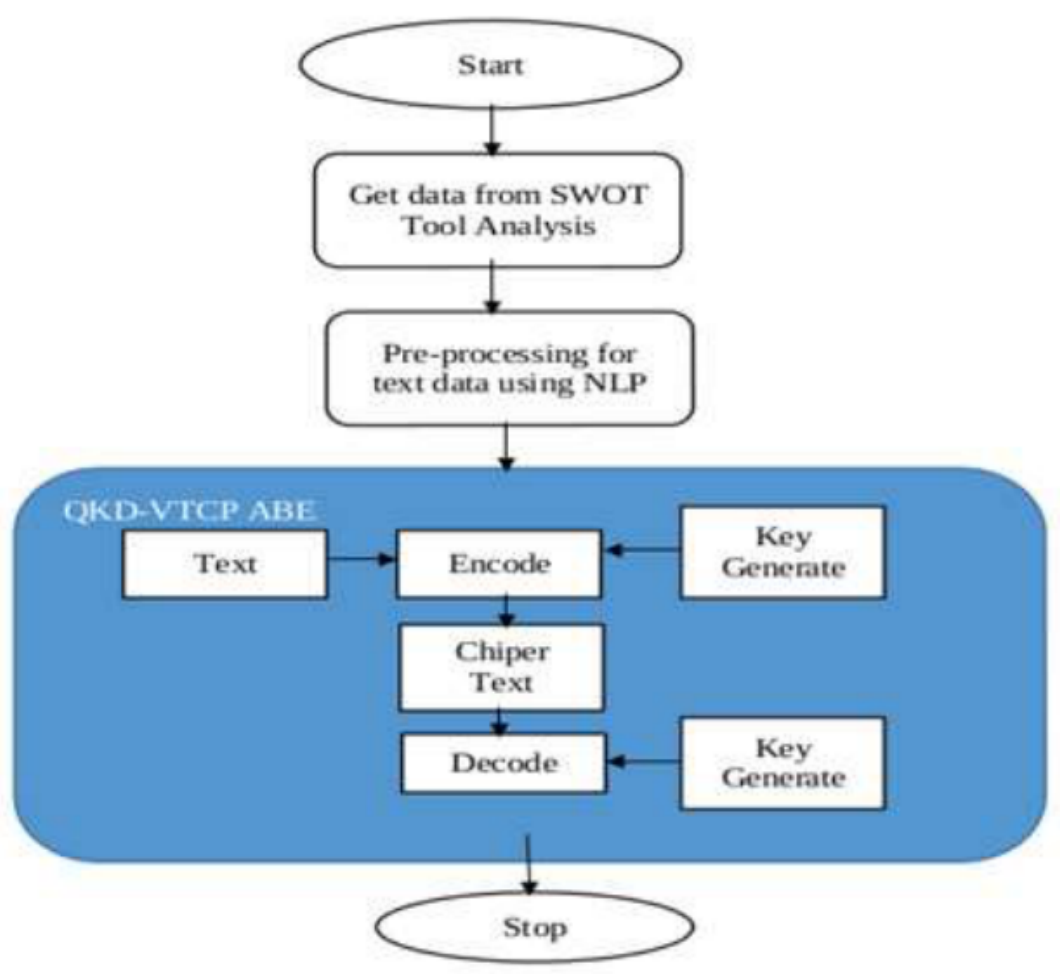

Figure.1: PROPOSED SYSTEM

\subsection{Quantum Key Distribution}

The figure 2 below illustrates the elemental model of a QKD includes two parties, one referred to as Alice and the other referred to as Bob. Who intend to exchange the key with an access to the communication channel which is public as well as a quantum communication channel? Even the eavesdropper is assumed to have an access to both the channels. The first QKD protocol BB84 which was based on the uncertainty principle of Heisenberg, was developed by Charles Bennett and Gilles Brassard in 1984. The fundamental idea of this protocol was that Alice will be able to transfer a secret key that is random to Bob with the help of a string of photons. The polarization of these photons encodes the bits of the secret keys. The heisenberg's principle of uncertainty ensured that eavesdropper was unable to measure the photon and transfer them to Bob without causing any disruption to the state of the photon hence revealing her existence. 


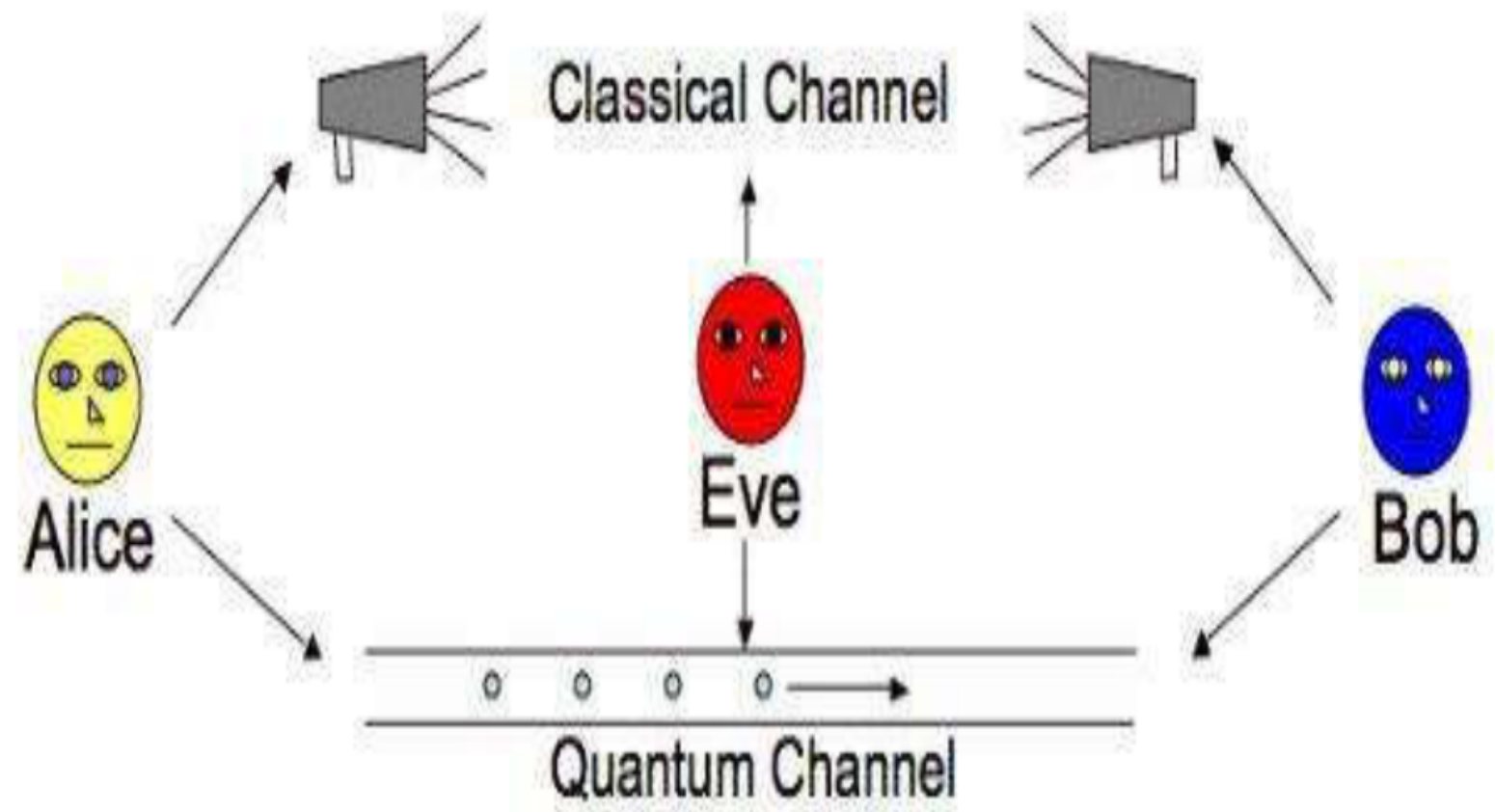

Figure 2. Model of QKD

The figure 3 below shows the bit encoding process when the photon is in the state of polarisation in the BB84 protocol. The binary 0 is defined as $0^{\circ}$ in the case of rectilinear bases and $45^{\circ}$ in case of diagonal bases. Similarly the binary 1 is defined as $90^{\circ}$ in case of rectilinear bases while it is defined as $135^{\circ}$ for diagonal bases. Hence the bit can be characterised by the polarisation of the photons on any one of the bases.

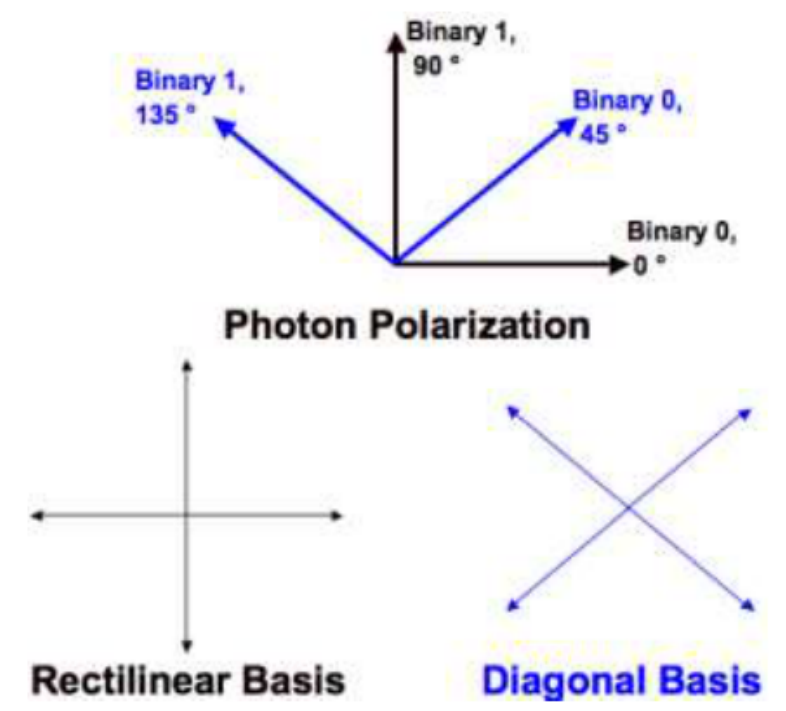

Figure 3. Bit encoding of BB84

In the first stage Alice will use a quantum channel for communication with Bob. Alice will begin the communication by selecting string of bits which is random and for every bit the basis for encoding the bit is selected randomly by alice itself. She will transfer photon of every bit along with the equivalent polarisation to Bob. For each of the photon received by him, Bob will assess the polarisation of the photon by randomly selected basis. Suppose Bob selects the same basis as that of Alice then Bob will be able to ascertain the bit sent by Alice correctly else the bit read by Bob will be random. In the second stage Alice will be alerted by Bob about any of the insecure channel, the basis utilised by for assessing the photon. Alice will revert back to 
Bob about his selection of basis for the photons. The bits which Bob assessed with different basis will be discarded by both of them provided errors are absent and manipulation of the photons is not done by anyone. The same string of bits which both of them will have is called sifted key. Before the process is completed Bob and Alice, for ensuring consistency, concur upon a bit subset. If these bits agree they are rejected and the rest of the bits will be used in forming the secret key which can be shared.

\section{CIPHERTEXT POLICY ATTRIBUTE-BASED ENCRYPTION (CP - ABE)}

The functional role of the keys and identities are generalised using the ABE schemes. In the case of ABE both the private as well as the public keys are replaced by the set of attributes. Furthermore $\mathrm{ABE}$ does not have certificates and the credentials of the cryptography are granted by an attribute authority which a trusted central party. The $\mathrm{CP}$ - ABE is a specific variant which links the attributes of the encryption process to the policies of the attributes. The encryption algorithm will encrypt the message for production of a ciphertext in such a way that the receiver which posses the attributes satisfies the policies of the attributes and is capable of decrypting the message (Weber, 2013).

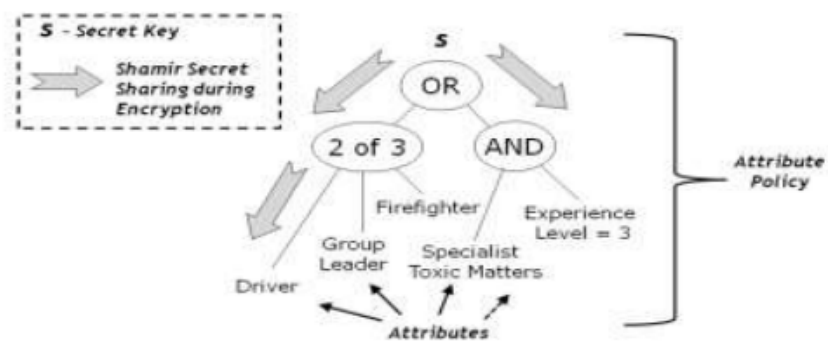

Figure 4. Policy for $\mathrm{CP}$ - ABE

For practical applications the $\mathrm{CP}$ - $\mathrm{ABE}$ is utilised as a hybrid encryption where the message is encrypted using a symmetric key which is selected randomly. Just this session key is then encrypted under the policy. Figure 4 illustrates the policy of CP - ABE consisting of attributes which are obtained from the domain of emergency management and it application in encrypting in the hybrid mode.

\section{RESULTS AND DISCUSSION}

The data is collected from the Indian treasury website and is stored as a txt document so that it can be read using the read command. The below figure 5 shows the text stored as .txt form which is read using the read command. 


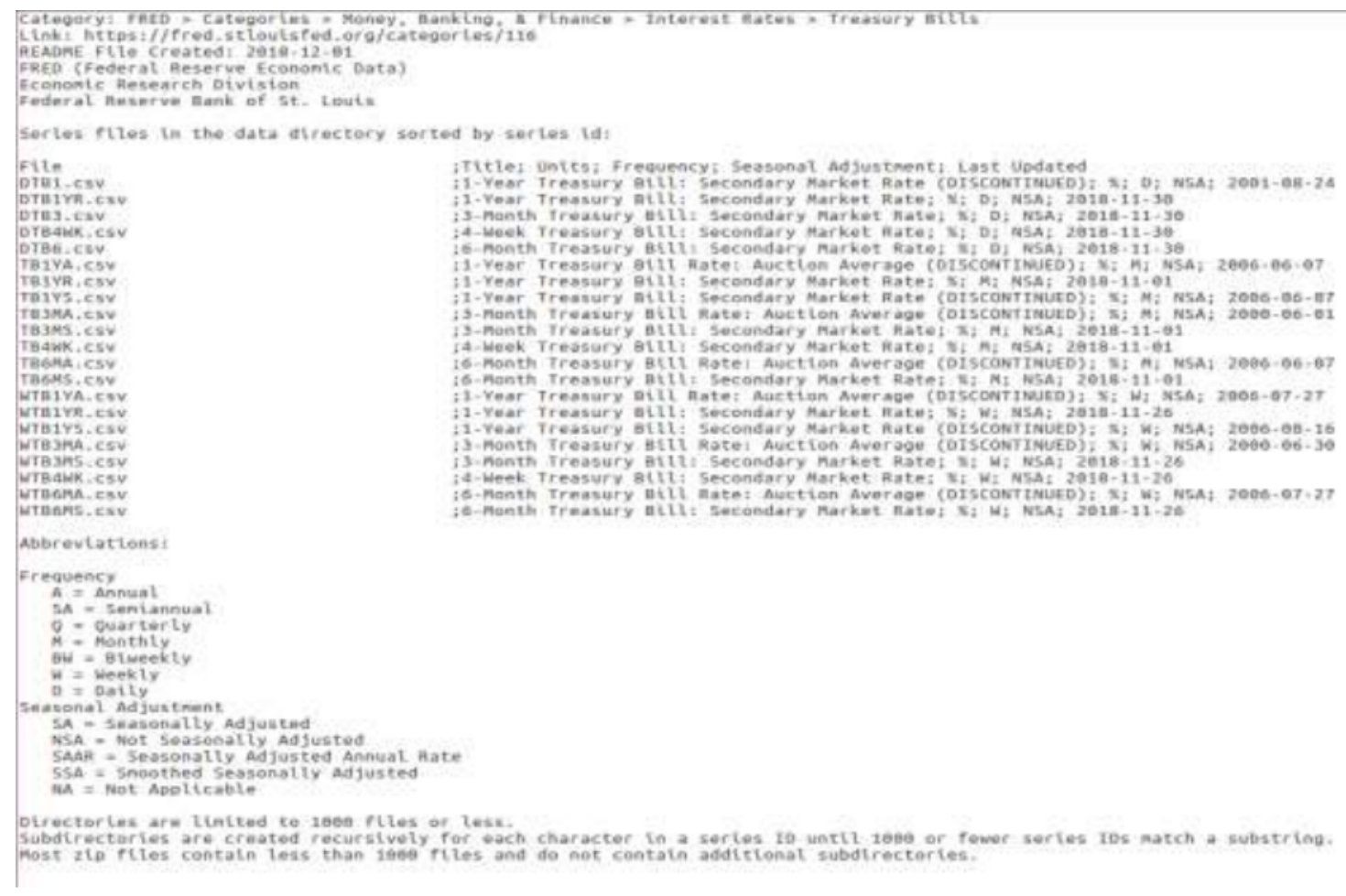

Figure 5. Input data

There are various unwanted characters like punctuations, special characters in the data which is removed and the data is arranged in a suitable manner using the natural language processing toolkit. The natural language programming toolkit is an advanced feature of the python programming language for preprocessing the data. Figure 6 shows the snapshot of the preprocessed data.

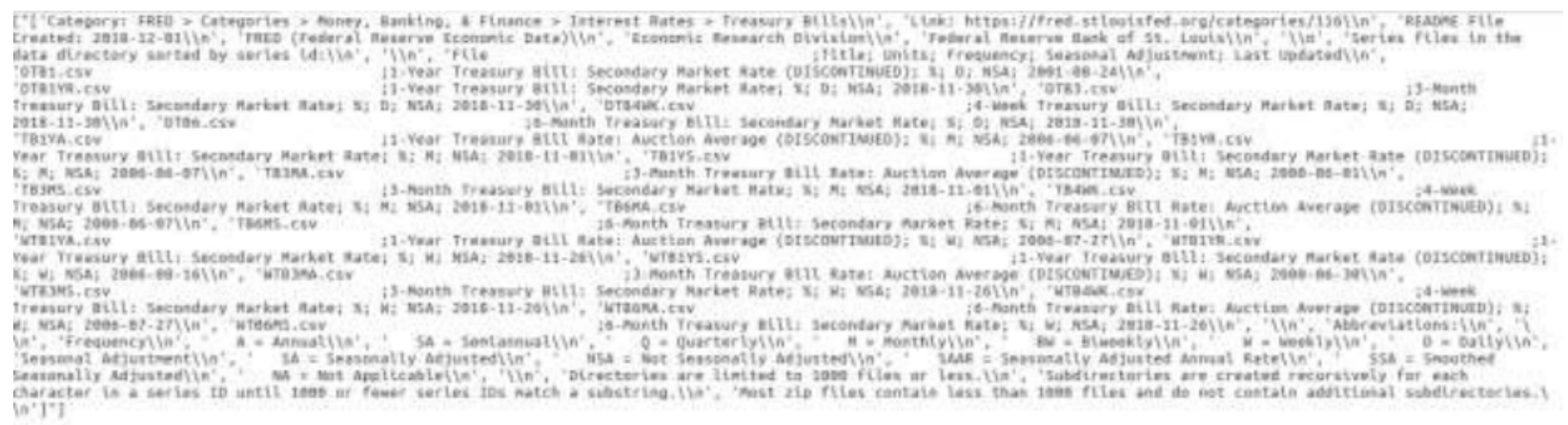

Figure 6. Preprocessed data

This preprocessed data is then given as an input to the ciphertext attribute based encryption where QKD technique is used for generating the keys. A public key for encrypting as well as decrypting the data is generated using the quantum key distribution technique. It is a protected and specialized technique whose uniqueness lies in the ability of two users communicating with each other to detect a third party who is trying to gain knowledge about the key. The proposed algorithm uses BB84 QKD protocol which is the first quantum cryptographic protocol. The snapshot in figure 7 shows the key generated. 


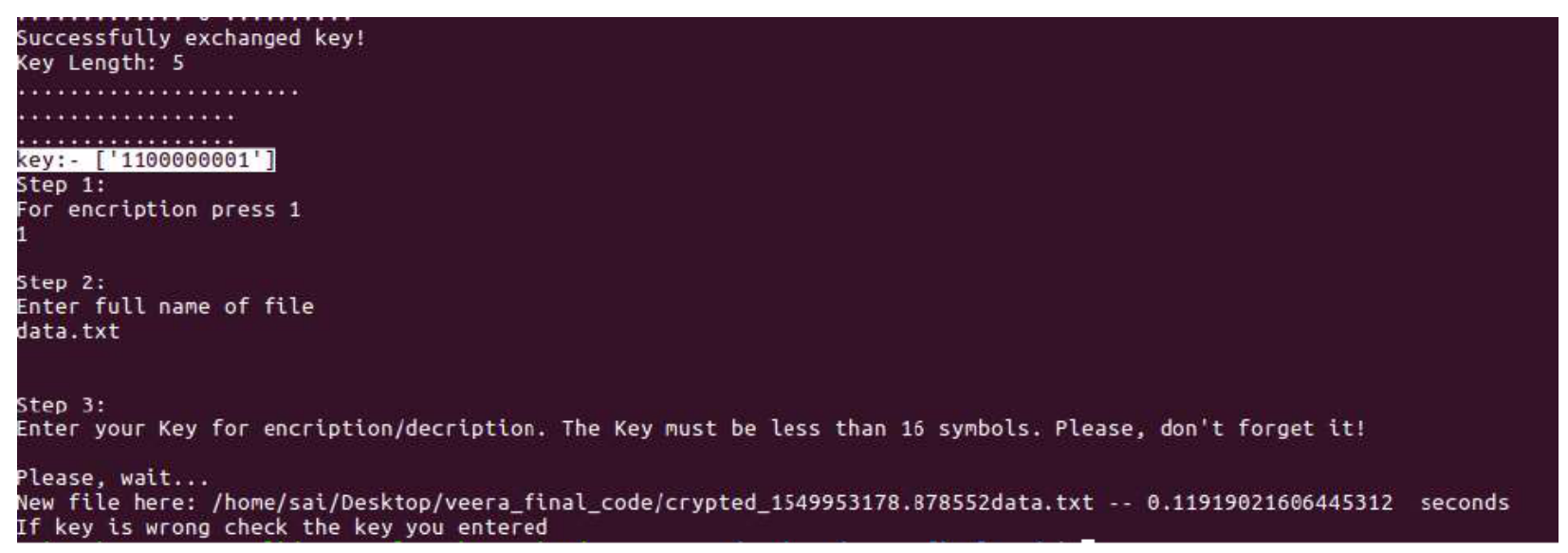

Figure 7. Key generation

Once the key is generated using QKD the private key is saved and the advanced $\mathrm{CP}$ - ABE technique is applied to encrypt the data for secure transfer from Alice to Bob supporting a block length of 128 bits and key lengths of 128 bits, 192 bits, and 256 bits. The figure 8 below shows the data which has been converted into cipher text.

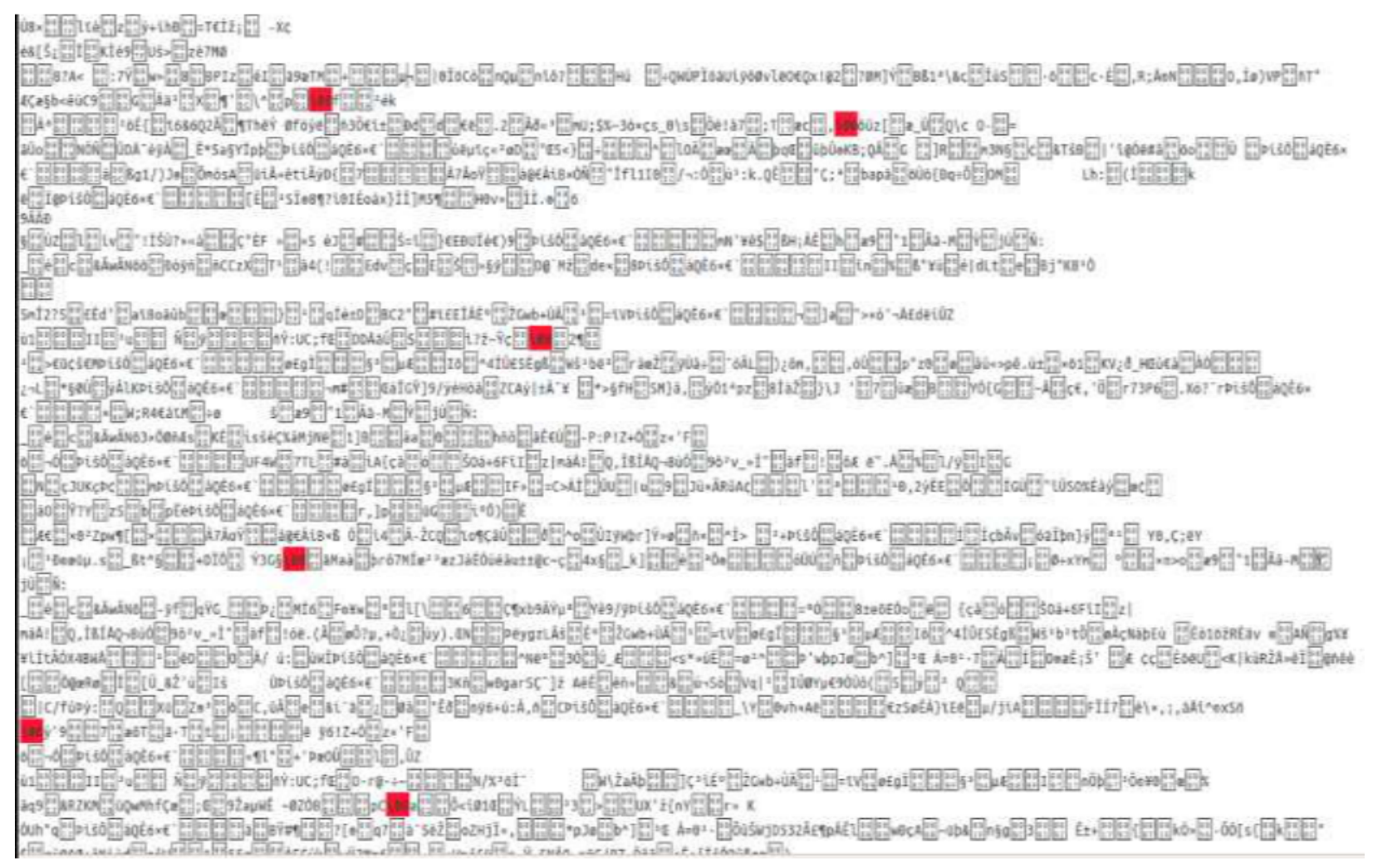

Figure 8. Encrypted data converted to cipher text

Once the data is converted to cipher text the same which was generated initially has to be entered again to decrypt the data and obtain it in its original form i.e. the same as the input. It can be observed in the figure 9 below that the same key generated initially is entered. 


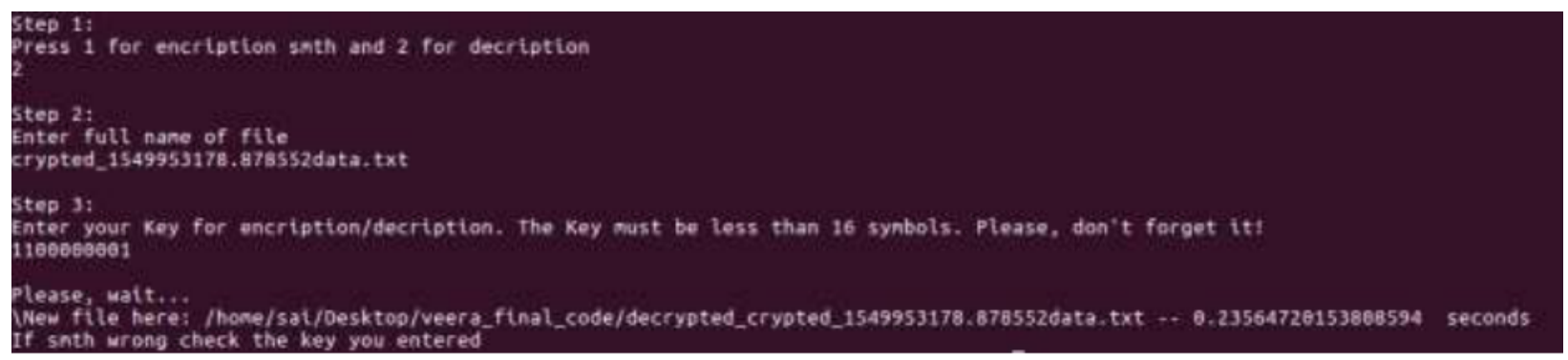

Figure 9. Decryption of the data by entering the same key generated in figure 7

The figure 10 below shows the snapshot of the final data which is obtained after decryption. This data will be nothing but the input data.

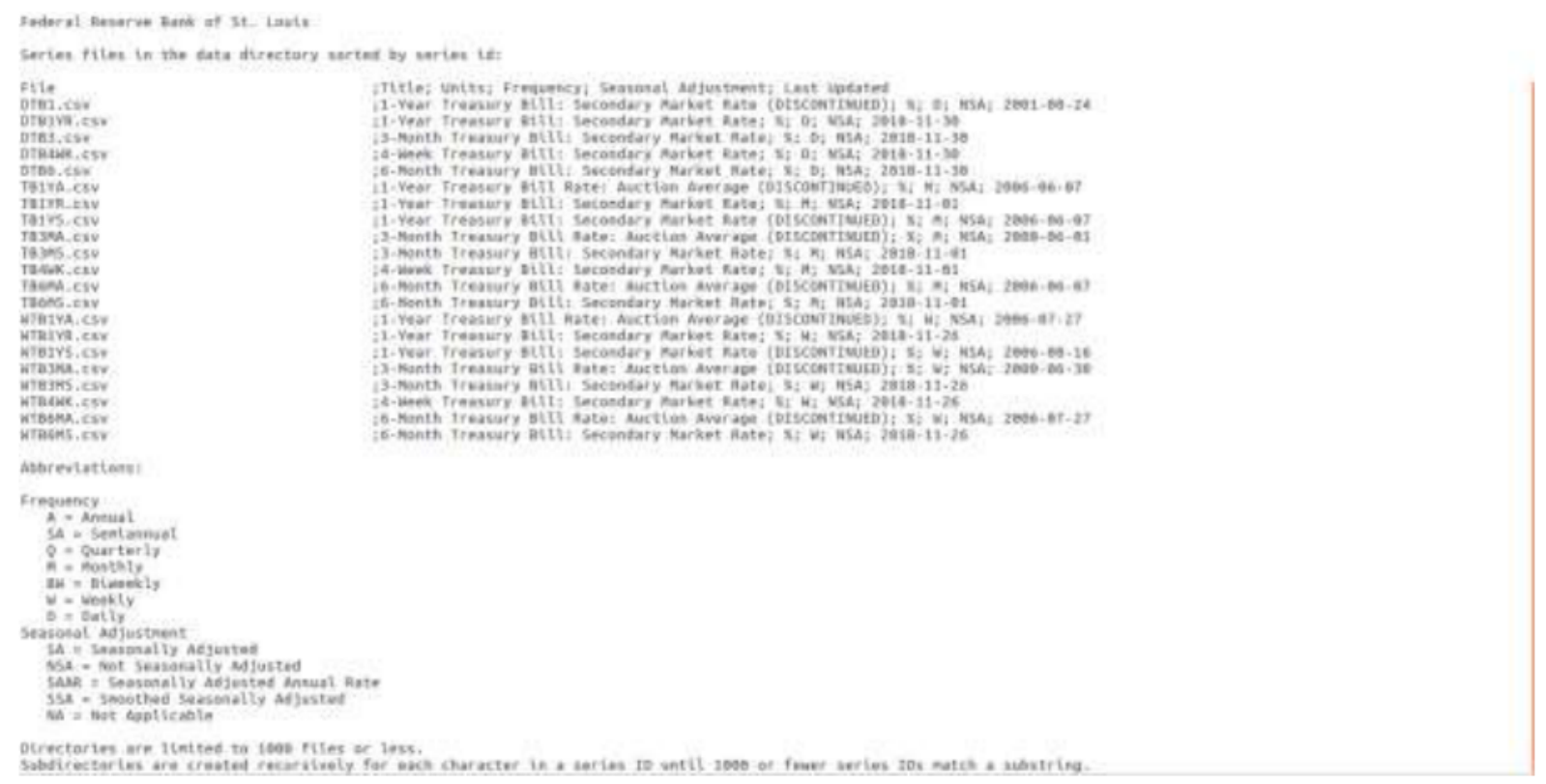

Figure 10. Final data obtained after decryption

\section{CONCLUSION}

This paper developed a security model for securing the treasury information system in India using the Quantum Key Distribution based verifiable and traceable cipher text attributes based encryption (QKD-VTCP ABE) technique. The exactness of the returned cipher text has been effectively verified using the proposed model and the extensive experiments emphasize that the proposed VTCP - ABE scheme effectively achieved traceability, verifiability and huge attribute universe.

\section{REFERENCES}

[1] Hla, D., \& Teru, S. P. (2015). Efficiency of Accounting Information System and Performance Measures-. Int. J. of Multidisciplinary and Current research, 3.

[2] Dandago, K. I., \& Rufai, A. S. (2014). Information technology and accounting information system in the nigerian banking industry. Asian Economic and Financial Review, 4(5), 655670.

[3] Anderson, D., Wu, R., Cho, J. S., \& Schroeder, K. (2015). Introduction: Global Challenges in Turbulent Times: Road to Sustainable E-government. In E-Government Strategy, ICT and Innovation for Citizen Engagement (pp. 1-10). Springer, New York, NY. 
[4] Kumar, T. V. (2015). E-governance for smart cities. In E-governance for smart cities (pp. 1-43). Springer, Singapore.

[5] Linders, D. (2012). From e-government to we-government: Defining a typology for citizen coproduction in the age of social media. Government Information Quarterly, 29(4), 446454.

[6] Malhotra, H., Bhargava, R., \& Dave, M. (2017, November). Challenges related to information security and its implications for evolving e-government structures: A comparative study between India and African countries. In Inventive Computing and Informatics (ICICI), International Conference on (pp. 30-35). IEEE.

[7] Sahai, A., \& Waters, B. (2005, May). Fuzzy identity-based encryption. In Annual International Conference on the Theory and Applications of Cryptographic Techniques (pp. 457-473). Springer, Berlin, Heidelberg.

[8] Bethencourt, J., Sahai, A., \& Waters, B. (2007, May). Ciphertext-policy attribute-based encryption. In Security and Privacy, 2007. SP'07. IEEE Symposium on (pp. 321-334). IEEE.

[9] Rouselakis, Y., \& Waters, B. (2013, November). Practical constructions and new proof methods for large universe attribute-based encryption. In Proceedings of the 2013 ACM SIGSAC conference on Computer \& communications security(pp. 463-474). ACM.

[10] Li, Q., Zhu, H., Ying, Z., \& Zhang, T. (2018). Traceable Ciphertext-Policy Attribute-Based Encryption with Verifiable Outsourced Decryption in eHealth Cloud. Wireless Communications and Mobile Computing, 2018.

[11] Li, L., Gu, T., Chang, L., Xu, Z., Liu, Y., \& Qian, J. (2017). A Ciphertext-Policy AttributeBased Encryption Based on an Ordered Binary Decision Diagram. IEEE Access, 5, 1137 1145.

[12] Canard, S., Phan, D. H., Pointcheval, D., \& Trinh, V. C. (2018). A new technique for compacting ciphertext in multi-channel broadcast encryption and attribute-based encryption. Theoretical Computer Science, 723, 51-72.

[13] Selar, G. D., \& Apoorva, P. (2017, June). Comparative study on KP-ABE and CP-ABE algorithm for secure data retrieval in military network. In Intelligent Computing and Control (I2C2), 2017 International Conference on (pp. 1-4). IEEE.

[14] Sangeetha, M., \& Vijaya Karthik, P. (2017, March). To provide a secured access control using combined hybrid key-ciphertext attribute based encryption (KC-ABE). In Intelligent Techniques in Control, Optimization and Signal Processing (INCOS), 2017 IEEE International Conference (pp. 1-4). IEEE.

[15] Chaudhari, S. H., \& Mandre, B. R. (2016). Secure data retrieval based on attribute-based encryption in cloud. International Journal of Computer Applications, 134(13).

[16] Haitjema, M. (2007). A survey of the prominent quantum key distribution protocols.

[17] Weber, S. G. (2013). Enabling End-to-End Secure Communication with Anonymous and Mobile Receivers-an Attribute-Based Messaging Approach. IACR Cryptology ePrint Archive, 2013, 478. 